\title{
Onset of illusory figures attenuates change blindness
}

\author{
Geoff G. Cole, Gustav Kuhn, And Simon P. Liversedge \\ University of Durham, Durham, England
}

\begin{abstract}
We examined whether the onset of a new object defined by illusory contours is detected with greater frequency than offset when neither is associated with a unique sensory transient. Observers performed a "one-shot" change detection task in which offsetting or onsetting elements of high luminance contrast circles generated the appearance or disappearance of a Kanizsa figure. Presenting "illusory figures" via this "flicker" method ensures that (1) any unique luminance transients associated with the two types of change are eliminated, and (2) the objects themselves can only be represented at a relatively high level. Results showed that offsets were detected more frequently than onsets only when they generated the onset of a Kanizsa figure. We argue that object appearance dominates object disappearance via mechanisms that operate at the level at which objects are constructed.
\end{abstract}

One of the primary functions of the human visual system is to alert the observer to sudden changes in the environment, as such changes could potentially be hazardous to the individual. Vision researchers have therefore been interested in the types of stimuli that accrue processing priority. Within this context, a large body of work has examined the relative effectiveness with which onsets accrue such priority relative to offsets. Since the onset of an object is more likely to pose a survival threat than an offset, one might expect appearance to be more effectively detected than disappearance. Yantis and Jonides's (1984) seminal study examined the issue by assessing whether a target associated with onsetting elements accrue shorter reaction times than a target associated with offsetting elements. Their displays began with the presentation of a number of figure-8 "placeholders" for $500 \mathrm{msec}$. Segments of the figures then disappeared to form letters. Simultaneously, an onset letter occurred at a previously unoccupied location and the target would either be the onset item or one of the letters generated by the offsetting elements of the figure-8s. Yantis and Jonides found that when the target coincided with the onset, reaction time (RT) was not affected by the number of search items. This suggests that the onsets were prioritized over offsets.

However, when examining the relative efficacy of onsets and offsets it is important to control for the amount of sensory change occurring at both types of stimulus. Miller (1989) argued that the onsets in Yantis and Jonides's study were associated with greater change than the offsets. He showed that when a larger number of offsetting elements occur at the placeholders no onset effect is observed (see also Martin-Emerson \& Kramer, 1997; Watson \& Humphreys 1995). In order to equate for visual change, the onsetting and offsetting stimuli should ideally be identi- cal. For instance, Pratt and McAuliffe (2001) presented observers with a visual cue followed by a target that occurred at the location of the cue on half the trials or in the contra lateral field on the other half. The cue was either the onset or offset of a small disc and thus was relatively well matched across both conditions. Pratt and McAuliffe showed that onsets have priority only when targets must be localized in space; when observers were required to decide whether a target was simply present, irrespective of location, no onset priority occurred. Similarly, Theeuwes (1991) also matched cue type in both onset and offset conditions by presenting either the onset or offset of a bar marker that occurred among an array of letters, one of which was the target. Results showed that although a target letter presented adjacent to a cue was prioritized, this priority was no greater for onsets than for offsets.

Presenting identical onsets and offsets will not in itself, however, guarantee parity of sensory change. For instance, forward luminance masking can occur at the location of offsetting stimuli such that the offset will be perceived to exist for a short duration despite its physical disappearance (see Gellatly, Cole \& Blurton, 1999; Gellatly \& Cole, 2000; Gibson, 1996; and Yantis \& Jonides, 1996). Recently, two methods have been reported that provide definitive control of any differences in sensory change. In a variation of Yantis and Jonides's (1984) procedure, Franconeri, Hollingworth, and Simons (2005) included a large moving "occluder" that passed over the array of figure-8s briefly concealing each. When these "old" objects reappeared (as letters), the additional (new) item occurred. Crucially, therefore, the new object had appeared in exactly the same manner as the old objects had reappeared. Indeed, the large luminance/motion signal that occurred across the display as a result of the occluder's

G. G. Cole, g.g.cole@durham.ac.uk 
movement would have masked the relatively small transient changes that occurred as a result of the new item appearing and figure-8s changing. Using this procedure, Franconeri et al. reported that targets associated with the new onset item did not accrue any greater priority relative to targets associated with one of the figure- $8 \mathrm{~s}$. The authors concluded therefore that onsets are not prioritized in the absence of an accompanying luminance transient.

The second method for presenting/changing items while controlling for sensory change is to briefly (e.g., $250 \mathrm{msec}$ ) offset all the old items. When they reappear the additional (new) item appears. As with the occluder procedure, the new object is therefore generated in an identical manner to that of old objects reappearing. This "flicker" procedure is, in effect, analogous to the change detection paradigm (e.g., Rensink, O'Regan, \& Clark, 1997; Simons, 1996; Simons $\&$ Rensink, 2005) in which a scene is presented, disappears, and reappears. Recently, Cole and his colleagues (e.g., Cole, Kentridge, \& Heywood, 2004; Cole, Kentridge, Gellatly, \& Heywood, 2003; Cole \& Liversedge, 2006) have adopted this technique, using it to examine which of the many events (e.g., color change, object onset) that occur in the visual environment is least susceptible to "change blindness." Cole et al. argued that relative detectability of different events within this paradigm provides an index of processing priority and found that the appearance of a new object was more resistant to change blindness compared with, for instance, objects that change color.

In the present experiment, we employed the flicker method in order to test whether onsets attenuate change blindness relative to offsets. A display was presented for a short time, disappeared and reappeared. Participants were then required to indicate whether a change occurred on the left or right side of the display. Cole et al. (2003; see also Phillips \& Singer, 1974; Stelmach, Bourassa, \& DiLollo, $1984)$ have previously reported that onsets are less susceptible to change blindness relative to offsets. Critically however, in the present experiment offsets created the onset of an object and conversely onsets created the offset of an object. This was achieved by offsetting or onsetting segments of circles to form either the appearance or disappearance of a Kanizsa square (see Figure 1A). Thus, uniquely, our new object, was not defined by fully specified constituent features. The perception of Kanizsa figures occurs despite the lack of a complete luminance contour. Instead, the figure is defined by "illusory" contours that the perceiver must visually interpolate (Gold, Murray, Bennett, \& Sekuler, 2000). Indeed, the perceptual interpolation of contours necessary for Kanizsa square identification requires processing to a level beyond basic visual processing associated with the identification of normal, fully specified, visual objects for which all constituent features are present. In effect, we directly pitted physical offsets that created the onset of a higher order object with physical onsets that created the offset of a higher order object. If increased detection for object appearance holds for objects that require perceptual interpolation of contours at a relatively high level, then visual offsets that lead to the creation of a new Kanizsa object should be detected more frequently than onsets. Put another way, visual onsets that usually confer increased rates of detection (Cole et al., 2003) will no longer do so if they lead to the removal of a Kanizsa square. In order to assess whether any detection differences are due to the creation of a Kanizsa figure, we included a control condition in which circle segments were orientated such that no Kanizsa figure was generated.

\section{METHOD}

On each trial of the experiment, 216 black $\left(0.5 \mathrm{~cd} / \mathrm{m}^{2}\right)$ circles were presented against a white $\left(81.5 \mathrm{~cd} / \mathrm{m}^{2}\right)$ background (see Figure 1A). These were arranged as nine columns of 12 , either side of a fixation point. The circles were $0.98^{\circ}$ in diameter and formed a rectangle measuring approximately $15^{\circ}$ in height and $21^{\circ}$ in width. At a number of locations in the display four segments of adjacent circles were absent in order to form between 9 and 12 Kanizsa squares. This occurred on half of the trials. On the other half these segments were positioned randomly such that no Kanisza squares were formed. As noted, these trials were included as a control to ensure that any facilitation for the detection of the Kanizsa squares was due to the detection of such a figure rather than increased detection for offsetting segments. The location of the segments was chosen pseudorandomly with the restriction that at least four of these segment groups would occur on either side of fixation. There was no relation between the number of objects on each side of the display and the side on which the change occurred. In order to ensure that our Kanizsa figures were indeed illusory objects we asked ten observers, who did not take part in the experiment proper, to view our displays and make judgments on two critical aspects of the stimuli. Kanizsa figures can be defined in two related ways concerning illusions of brightness and contour (Frisby, 1979). The area within the figure appears brighter than surrounding areas and illusory contours appear to form the straight edges of the figure. We asked our observers whether the area within the squares was brighter, dimmer, or no different than the surrounding areas. Then the observers were shown an example of a classical Kanizsa figure (from Frisby, 1979) and asked whether our figures did or did not induce the illusory contours. Nine of the ten observers perceived the area within our illusory figures as brighter than the surround, and all ten perceived illusory contours. This therefore demonstrates that our stimuli were indeed illusory figures constructed at a relatively high level.

For the experiment proper, a $2 \times 2$ within-participants design was employed. The first factor was the orientation of the circle segments and the second factor was either the onset or offset of the segments. Each trial began with the presentation of a fixation point for $1,500 \mathrm{msec}$ before the appearance of the first image for $250 \mathrm{msec}$. A uniform blank white frame then appeared for $250 \mathrm{msec}$ followed by the appearance of the second image. This image remained until the participant responded. Participants were required to indicate whether a change had occurred on the left or right side of fixation. They were invited to guess if they did not detect any change. The beginning of a trial was initiated by the participant's response on the previous trial. Participants were asked to maintain fixation for the duration of each trial and that accuracy rather speed was important. They were not told what types of change would occur except that a single change occurred across the two images. For every trial on which there was an onset of circle segments, the same pair of images was repeated in reverse order, thus creating an offset of circle segments. This ensured that over the whole experiment onsets and offsets occurred at the same locations and thus were equally perceptible. Each participant was given 2 blocks of one hundred and twenty eight trials. The orientation of segments factor was blocked and presentation order counterbalanced. The onset-offset factor was run within block and trial types presented randomly. Twenty practice trials were given following a demonstration trial. The experiment was carried out in a well-lit room and was driven by a Pentium PC linked to a standard color monitor running at $60 \mathrm{~Hz}$. Participants 
A Circle Segments Orientated to Form Kanizsa Figures

Segment Offsets
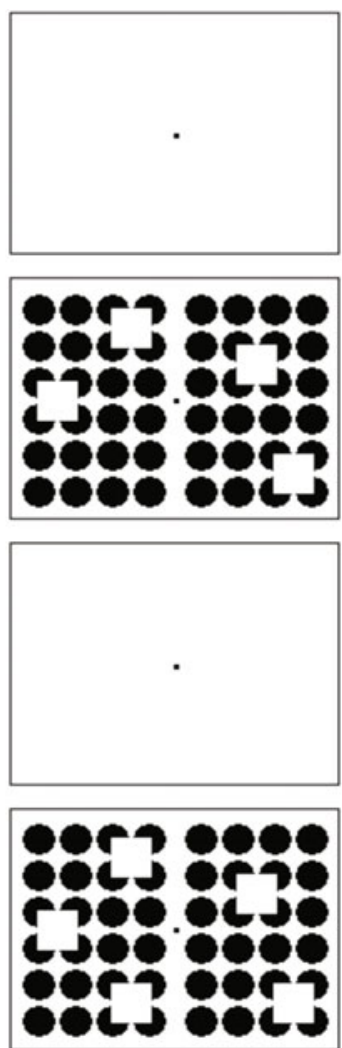

Segment Onsets
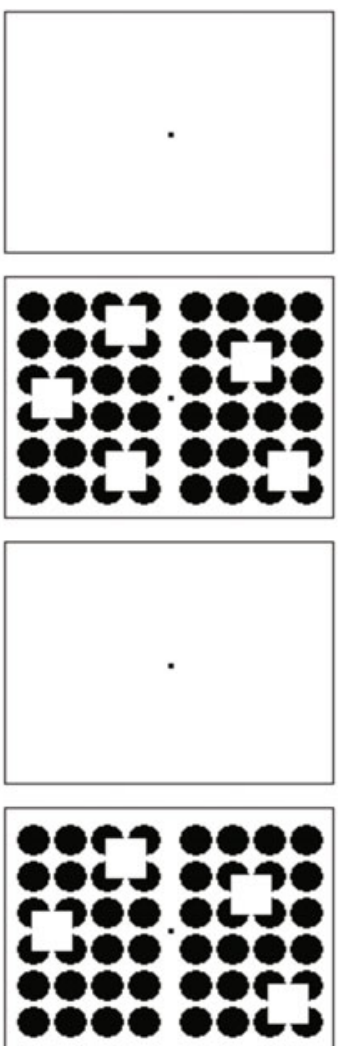

\section{Circle Segments Orientated Randomly}

Segment Offsets

Segment Onsets
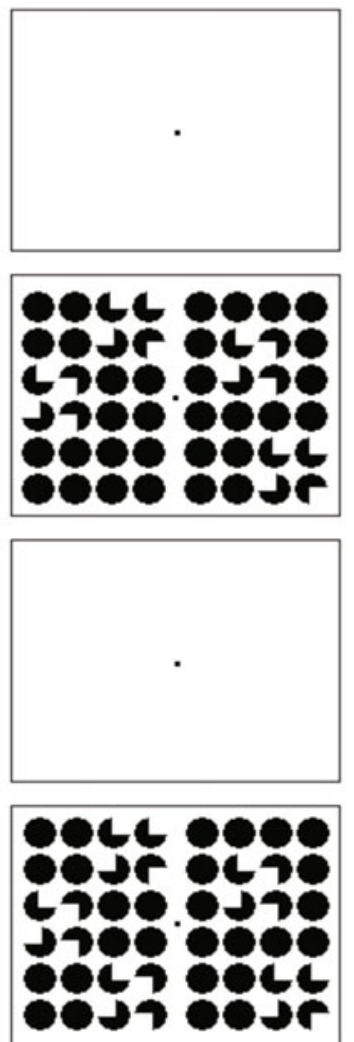


B

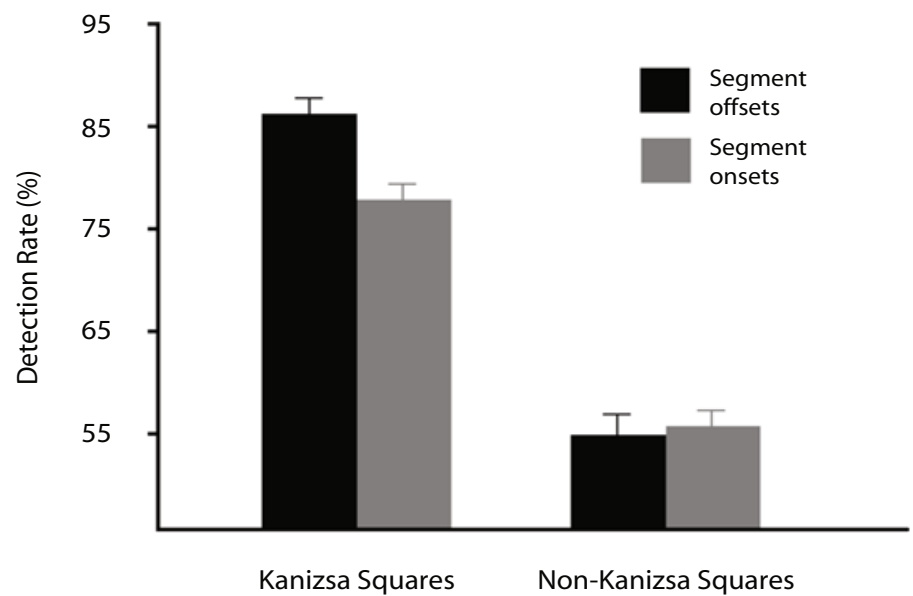

Figure 1. (A) Examples of the four conditions. Segments of the black circles either offset to form the onset of a Kanizsa square (far left panel) or onset to form its offset (left middle). On half the trials these segments were oriented such that no Kanizsa squares were formed (right panels). In each of the four examples shown here the change occurs at the bottom left of the display relative to the fixation point. Note that the circles and squares are not to scale. Two hundred sixteen circles also occurred in the displays rather than the 48 shown here. (B) Correct detection rates for either the onset or offset of circle segments. 
were seated approximately $70 \mathrm{~cm}$ from the display and responded by pressing either the forward slash or backward slash on a standard keyboard. There were 30 participants.

\section{RESULTS}

Figure 1B shows mean detection rates for segment onsets and offsets in both orientation conditions. Each participant's mean detection rate for each of the four conditions was entered into a $2 \times 2$ within-participants ANOVA. A significant main effect was observed for segment orientation such that changes to segments that were orientated to form Kanisza squares were detected more frequently than segments that did not form Kanisza squares $[F(1,29)=$ $\left.185, p<.01, \eta^{2}=.87\right]$. The main effect of onset/offset was also significant $\left[F(1,29)=9.7, p<.01, \eta^{2}=.25\right]$. Hence, offsetting segments were detected more frequently than onsetting segments. The crucial analysis however concerns the significant interaction $[F(1,29)=5.5, p<$ $\left..05, \eta^{2}=.16\right]$. The nature of this interaction is revealed by comparisons of performance for the detection of segment onsets and offsets for both orientation conditions. Enhanced detection of segment offsets relative to onsets only occurred when they formed the onset of a Kanizsa square $[t(29)=4.86, p<.01]$; segment offsets that did not form such a square were not detected with greater frequency than segment onsets $[t(29),<1]$. These results clearly show that visual offsets that result in the visual onset of an illusory Kanizsa square are less susceptible to change blindness than when counterpart visual onsets occur. Importantly, when visual offsets occur, but do not create a Kanizsa square onset, no difference in change detection performance was observed.

\section{DISCUSSION}

The current study examined the propensity with which visual onsets are detected relative to visual offsets within the change detection paradigm. Previous work has shown that onsets are less susceptible to change blindness compared with offsets (e.g., Cole et al., 2003; Cole et al., 2004; Cole \& Liversedge, 2006; Phillips \& Singer, 1974; Stelmach, Bourassa, \& DiLollo, 1984). Crucially however, the offsets presented in the present work created the onset of an object represented at a relatively high level while the onsets created the offset of such an object. This was achieved by the removal (or addition) of quarter segments from high luminance contrast circles to form either the appearance or disappearance of an illusory Kanizsa figure. If the visual system is particularly sensitive to the detection of objects - that is, stimuli that have been processed to a relatively high level, we should observe increased detection rates for offsets that create the appearance of an object. Results showed this to be the case; offsetting stimuli that produce the appearance of an illusory Kanizsa square attenuate change blindness. By contrast, offsetting stimuli that did not result in the appearance of a Kanizsa square, or onsetting stimuli that created the disappearance of such a square did not reduce change blindness. ${ }^{1}$
The fact that object onset facilitation occurred when offsetting stimuli resulted in the creation of a Kanizsa square demonstrates that the onset effect was not caused by a fully specified object as defined by a complete set of luminance contours. Instead, the effect occurred as a consequence of the onset of an illusory object that required the perceiver to interpolate illusory contours in order that it might be identified. That is to say, the effect holds for objects that require visual processing to a level beyond basic perception associated with the identification of objects that are fully specified in terms of their constituent luminance contours. Additionally, the transient associated with the new object was equal to that associated with changes to all other stimuli in the display. This was achieved via the "flicker" method commonly employed with the change detection procedure. We suggest therefore that for the detection of new onsets can be represented at the level at which objects are constructed.

An important aspect of the results concerns the mechanism responsible for the onset effect. Although the flicker procedure has ensured that low-level sensory change processing cannot account for the onset advantage, a central question is whether the effect relied on attentional mechanisms. Although attention clearly modulates the degree to which change blindness is attenuated (Rensink, O'Regan, \& Clark, 1997; Scholl, 2000) it does not necessarily follow that change detection performance reveals how attention was operating. Yantis and Egeth (1999) have shown that visual properties which are highly salient, in the sense that they pop-out from a visual display (Triesman \& Gelade, 1980), will not necessarily do so automatically, that is, when the observer is not set to search for the unique item. Thus, if a highly salient item does not necessarily attract attention, a change in a change detection task is unlikely to do so in a strong sense. The critical point however is that we have observed a facilitatory effect for object onset when sensory change was controlled. This supports work suggesting that appearance of objects receive processing priority (Cole, Kentridge, \& Heywood, 2005; Yantis \& Jonides, 1984). Moreover, the notion that relative degrees of change blindness can provide an index of processing priority has now been used by a number of researchers. For instance, Ro, Russell, and Lavie (2001) tested the hypothesis that since faces have ecological significance face changes should be less susceptible to change blindness compared with changes to common objects. Similarly, Pisella, Berberovic, and Mattingly (2004) have used the method to assess which visual attributes are least likely to go unnoticed in neglect patients. Such studies contrast the majority of change detection work that has been primarily concerned with what change blindness reveals about the limited representation of a visual scene. Furthermore, since our experiment involved the comparison of one image with a subsequent image, the effect may even have involved visual short-term memory.

Related to the question of mechanisms responsible for the onset advantage is the issue of how object symmetry contributed to the effect. In our Kanizsa figure onset/offset conditions the objects were partly created as a conse- 
quence of symmetry whereby the circle segments were oriented such that a square was formed. By contrast, in the control condition, where no Kanizsa figures were formed, there was no symmetry. Hence, not only was there an absence of an object but there was also an absence of symmetry. It is therefore possible that the onset detection advantage was actually an advantage for the appearance of symmetry with illusory contours playing little or no role. Similarly, our Kanizsa squares were partly generated by the grouping of four luminance-defined corners. Hence, the onset effect may also have been driven by a more general grouping process. However symmetry, grouping and objectness are clearly related. Indeed, preattentive construction of objects is commonly assumed to occur on the basis of gestalt principles of organization which include symmetry and grouping.

Finally, our results also demonstrate that facilitation for the appearance of new objects in a visual scene cannot be explained by changes in spatial configuration. Simons (1996) has shown that although much information within visual short-term memory is poorly represented, changes in the spatial configuration of objects are relatively well retained. Similarly, Jiang, Olson, and Chun (2000) showed that whereas changes in the spatial layout of a scene interferes with the detection of changes to the features of objects, changes in features do not impair spatial change detection. This leads to the possibility that the reason new objects accrue priority is that such onsets coincide with a change in spatial layout. The new onset effect might therefore occur as a consequence of facilitated spatial layout processing, with new onset facilitation being epiphenomenal. However, the change in spatial layout in both our Kanizsa onset and Kanizsa offset conditions was identical therefore ruling out this possibility.

\section{AUTHOR NOTE}

This work was supported by U. K. Economic and Social Research Council Grant RES-000-22-0888 to the first author and by U. K. Biotechnology and Biological Sciences Research Council Grant 12/S19168 to the third author. Correspondence concerning this article should be addressed to G. G. Cole, Department of Psychology, University of Durham, South Road, Durham DH1 3LE, England (e-mail: g.g.cole@ durham.ac.uk).

\section{REFERENCES}

Cole, G. G., Kentridge, R. [W.], Gellatly, A., \& Heywood, C. (2003). Dectectability of onsets versus offsets in the change detection paradigm. Journal of Vision, 3, 22-31.

Cole, G. G., Kentridge, R. W., \& Heywood, C. A. (2004). Visual salience in the change detection paradigm: The special role of object onset. Journal of Experimental Psychology: Human Perception \& Performance, 30, 464-477.

Cole, G. G., Kentridge, R. W., \& Heywood, C. A. (2005). Object onset and parvocellular guidance of attentional allocation. Psychological Science, 16, 270-274.

Cole, G. G., \& Liversedge, S. P. (2006). Change blindness and the primacy of object appearance. Psychonomic Bulletin \& Review, 13, 588-593.

Franconeri, S. L., Hollingworth, A., \& Simons, D. J. (2005). Do new objects capture attention? Psychological Science, 16, 275-282.

FrisBY, J. P. (1979). Seeing: Illusion, brain, and mind. Oxford: Oxford University Press.
Gellatly, A. R. H., \& Cole, G. G. (2000). Accuracy of target detection in new-object and old-object displays. Journal of Experimental Psychology: Human Perception \& Performance, 26, 889-899.

Gellatly, A. R. H., Cole, G. G., \& Blurton, A. (1999). Do equiluminant object onsets capture visual attention? Journal of Experimental Psychology: Human Perception \& Performance, 25, 1609-1624.

GiBson, B. S. (1996). The masking account of attentional capture: A reply to Yantis and Jonides (1996). Journal of Experimental Psychology: Human Perception \& Performance, 22, 1514-1520.

Gold, J. M., Murray, R. F., Bennett, P. J., \& Sekuler, A. B. (2000). Deriving behavioural receptive fields for visually completed contours. Current Biology, 10, 663-666.

JiAnG, Y., Olson, I. R., \& CHUN, M. M. (2000). Organization of visual short-term memory. Journal of Experimental Psychology: Learning, Memory, \& Cognition, 26, 683-702.

Martin-Emerson, R., \& Kramer, A. F. (1997). Offset transients modulate attentional capture by sudden onsets. Perception \& Psychophysics, 59, 739-751.

Miller, J. (1989). The control of attention by abrupt visual onsets and offsets. Perception \& Psychophysics, 45, 567-571.

Phillips, W. A., \& Singer, W. (1974). Function and interaction of on and off transients in vision: I. Psychophysics. Experimental Brain Research, 19, 493-506.

Pisella, L. P., Berberovic, N., \& Mattingly, J. P. (2004). Impaired memory for location but not for color or shape in visual neglect: A comparison of parietal and non-parietal lesions. Cortex, 40, 379-390.

Pratt, J., \& MCAuliffe, J. (2001). The effects of onset and offset on visual attention. Psychological Research, 65, 185-191.

Rensink, R. A., O'Regan, J. K., \& ClaRK, J. J. (1997). To see or not to see: The need for attention to perceive changes in scenes. Psychological Science, 8, 368-373.

Ro, T., Russell, C., \& Lavie, N. (2001). Changing faces: A detection advantage in the flicker paradigm. Psychological Science, 12, 94-99.

SCHOLL, B. J. (2000). Attenuated change blindness for exogenously attended items in a flicker paradigm. Visual Cognition, 7, 377-396.

Simons, D. J. (1996). In sight, out of mind: When object representations fail. Psychological Science, 7, 301-305.

Simons, D. J., \& Rensink, R. A. (2005). Change blindness: Past, present, and future. Trends in Cognitive Sciences, 9, 16-20.

Stelmach, L. B., Bourassa, C. M., \& Dilollo, V. (1984). Detection of stimulus change: The hypothetical roles of visual transient responses. Perception \& Psychophysics, 35, 245-255.

Theeuwes, J. (1991). Exogenous and endogenous control of attention: The effect of visual onsets and offsets. Perception \& Psychophysics, 49, 83-90.

Treisman, A., \& Gelade, G. (1980). A feature-integration theory of attention. Cognitive Psychology, 12, 97-136.

Watson, D. G., \& Humphreys, G. W. (1995). Attention capture by contour onsets and offsets: No special role for onsets. Perception \& Psychophysics, 57, 583-597.

Yantis, S., \& Egeth, H. E. (1999). On the distinction between visual salience and stimulus-driven attentional capture. Journal of Experimental Psychology: Human Perception \& Performance, 25, 661-676.

YANTIS, S., \& Jonides, J. (1984). Abrupt visual onsets and selective attention: Evidence from visual search. Journal of Experimental Psychology: Human Perception \& Performance, 10, 601-621.

YANTIS, S., \& Jonides, J. (1996). Attentional capture by abrupt onsets: New perceptual objects or visual masking? Journal of Experimental Psychology: Human Perception \& Performance, 22, 1505-1513.

\section{NOTE}

1. One might assume that segment onsets would accrue increased detection relative to segment offsets in the random orientation condition. However, Cole et al. (2004) provided strong evidence that attenuated change blindness for onsets only occurs for onset objects rather than onsets that add to an existent object.

(Manuscript received April 5, 2006; revision accepted for publication November 20, 2006.) 